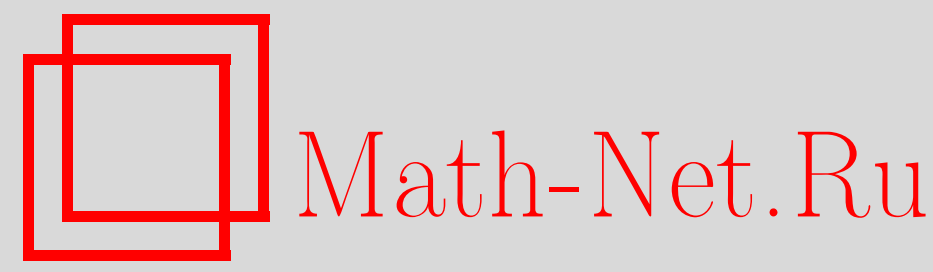

Ю. С. Семенов, Доказательство обобщенной гипотезы Х. Нейман для некоторых почти свободных групп, Матем. сб., 1996, том 187, номер 10, 145-160

DOI: https://doi.org/10.4213/sm169

Использование Общероссийского математического портала Math-Net.Ru подразумевает, что вы прочитали и согласны с пользовательским соглашением http://www . mathnet.ru/rus/agreement

Параметры загрузки:

IP: 35.174 .16 .151

26 апреля 2023 г., 15:36:47 
УДК 512.54

\author{
Ю.С. Семенов
}

\title{
Доказательство обобщенной гипотезы Х. Нейман для некоторых почти свободных групп
}

\footnotetext{
В работе доказана обобщенная гипотеза Х. Нейман для групп, содержащих свободное произведение трех циклических групп второго порядка в качестве подгрупшы конечного индекса.
}

Библиограффия: 3 названия.

\section{Введение}

Пусть $G$ - некоторая конечно порожденная неэлементарная (т.е. не почти циклическая) группа, содержащая свободную подгруппу $G_{0}$ конечного индекса. Обозначим через $[H]$ класс сопряженности подгруппы $H$ в группе $G$. Рассмотрим свободный $\mathbb{Z}$-модуль $D(G)$ с базисом, состоящим из всех $[H]$, где $H$ - бесконечная конечно порожденная подгруппа в $G$. В [1] показано, что на $D(G)$ можно задать такую структуру коммутативного ассоциативного кольца с единицей, что для любых $[H],[K] \in D(G)$

$$
[H][K]=\sum_{g \in(H \backslash G / K)^{*}}\left[H \cap K^{g}\right] .
$$

В этой сумме представители $H, K$ соответствующих классов сопряженности $[H],[K]$ фиксированы, а суммирование производится по всем представителям $g$ двойных смежных классов $H g K \in H \backslash G / K$, для которых подгруппы $H \cap g K g^{-1}$ бесконечны (таких классов конечное число, и можно доказать, что $H \cap g K g^{-1}$ будет конечно порожденной подгруппой в $G)$.

Для любого класса $[H] \in D(G)$ положим

$$
r([H])=\chi(H) / \chi(G)
$$

где $\chi(L)$ - эйлерова характеристика групшы $L$, и продолжим $r$ по линейности на все кольцо $D(G)$. Функция $r$ называется приведенной әйлеровой характеристикой. Будем говорить, что для групшы $G$ выполнено неравенство $X$. Нейман, если

$$
r([H][K]) \leqslant\left|\chi\left(G_{0}\right)\right| r([H]) r([K])
$$

для любых $[H],[K] \in D(G)$ и для любой свободной подгрупшы конечного индекса $G_{0}$. Заметим, что множитель $\left|\chi\left(G_{0}\right)\right|$ всегда больше либо равен 1 в силу условий, наложенных на группу $G$, и можно считать в неравенстве $(0.1)$, что индекс не однозначно определенной подгруппы $G_{0}$ в группе $G$ минимален.

Работа вьполнена при частичной финансовой поддержке Российского фонда фундаментальных исследований (грант № 95-01-00733). 
В частности, если $G$ - свободная группа ранга 2 , то неравенство (0.1) можно переписать в виде

$$
\sum_{g \in(H \backslash G / K)^{*}}\left(\operatorname{rank}\left(H \cap g K g^{-1}\right)-1\right) \leqslant(\operatorname{rank}(H)-1)(\operatorname{rank}(K)-1)
$$

(мы опускаем квадратные скобки, означающие классы сопряженности), поскольку для любой конечно порожденной свободной групшы $F$ эйлерова характеристика равна $1-\operatorname{rank}(F)$.

Неравенство (0.2) известно как усиленный вариант гипотезы Х. Нейман, предложенный У. Нейманом в работе [2]. Сама же гипотеза была впервые сформулирована в [3] следующим образом: верно ли, что для двух нетривиальных конечно порожденных подгрупп $H$ и $K$ свободной группы $F$ (которую всегда можно считать ранга 2) выполнено неравенство

$$
\operatorname{rank}(H \cap K)-1 \leqslant(\operatorname{rank}(H)-1)(\operatorname{rank}(K)-1) .
$$

Ясно, что (0.3) получается из (0.2) рассмотрением одного слагаемого в сумме при $g=1$ (если $H \cap K$ - тривиальная подгруппа, то (0.3) очевидно) и что (0.1) является обобщением неравенства $(0.2)$ на цельй класс групп.

Это дает основание выдвинуть следующую гипотезу.

ОБОБШЕННАЯ ГИПОТЕЗА Х. НЕЙМАН. Для любой неэлементарной конечно порожденной почти свободной группы $G$ выполнено неравенство $Х$. Нейман.

Гипотеза Х. Нейман может быть сформулирована и для некоторых неэлементарных гиперболических групп (в смысле Громова), например, для фундаментальных групп поверхностей рода, большего единицы.

В данной работе обобщенная гипотеза Х. Нейман доказана для группы $\Gamma=(\mathbb{Z} / 2 \mathbb{Z}) *(\mathbb{Z} / 2 \mathbb{Z}) *(\mathbb{Z} / 2 \mathbb{Z})($ теорема 1$)$, а также для любой группы $G$, которая содержит Г как подгруппу конечного индекса (теорема 2).

Структура работы такова. В первой части собраны технические результаты (в основном известные), касающиеся некоторого класса размеченных графов. Под графом мы всегда будем подразумевать некоторьй локально конечный 1-комплекс $V$. Граф назовем размеченным, если имеются два отображения множества вершин $\mu_{0}: V^{0} \rightarrow M_{0}$ и множества ребер $\mu_{1}: V^{1} \rightarrow M_{1}$ в некоторые непересекаюшиеся множества меток $M_{0}$ и $M_{1}$.

Под путем р в графе $V$ понимается комбинаторный путь, т.е. последовательность ребер $e_{1} e_{2} \ldots e_{m}$ таких, что начало следуюшего ребра совпадает с концом предыдущего. Петля $p$ (замкнутый путь) назьвается минимальной, если среди пар $\left\{e_{k}, e_{k+1}\right\}$ и $\left\{e_{m}, e_{1}\right\}, k=1, \ldots, m-1$, нет пар взаимно обратных ребер.

Во второй части доказана обобщенная гипотеза X. Нейман для любой группы $G$, содержащей группу $\Gamma=(\mathbb{Z} / 2 \mathbb{Z}) *(\mathbb{Z} / 2 \mathbb{Z}) *(\mathbb{Z} / 2 \mathbb{Z})$ как подгруппу конечного индекса. В качестве приложения полученного результата приводится конструкция коммутативной унитальной банаховой алгебры $\widehat{A}(G)$ для такой групшы $G$. 


\section{§1. Подгруппы свободной группы и графы}

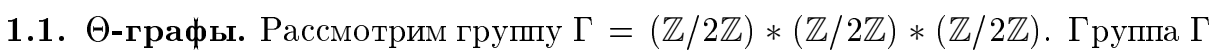
имеет очевидное копредставление $\left\langle x, y, z \mid x^{2}=y^{2}=z^{2}=1\right\rangle$, которым мы и будем в дальнейшем пользоваться. Зафиксируем систему порождаюших $S=\{x, y, z\}$ группы $\Gamma$.

Пусть $T=C(\Gamma, S)$ - неориентированньй граф Кэли для $Г$ относительно $S$, т.е. $T$ - это 1-комплекс, вершины которого находятся во взаимно однозначном соответствии с элементами $g \in \Gamma$, а неориентированные ребра - с неупорядоченными парами элементов $(g, h)$ таких, что $g^{-1} h \in S$. Заметим, что каждому ребру $e$, соединяющему $g$ и $h$, можно приписать метку $\mu(e)=g^{-1} h \in S$. Таким образом, метка ребра - это один из порождающих $x, y$ или $z$.

Хорошо известно, что $T$ - это симплициальное дерево, каждая вершина которого имеет степень 3 . Группа Г симплициально действует на $T$, сохраняя метки ребер, причем действие $Г$ на вершинах $T$ свободно и транзитивно. Стабилизатор ребра $e$ - циклическая группа второго порядка, сопряженная в Г с одной из подгрупा $\langle x\rangle,\langle y\rangle$ или $\langle z\rangle$ в зависимости от метки ребра $e$.

Дерево $T$ можно превратить в метрическое пространство таким образом, что расстояние $|g-h|$ между двумя вершинами $g, h$ (мы не делаем различия между вершинами $T$ и элементами группы $\Gamma$ ) равнялось бы длине кратчайшей записи $g^{-1} h$ в порождающих $S$, а каждое ребро $е$ было бы изометрично единичному интервалу $[0,1] \subset \mathbb{R}$. При этом Г будет группой изометрий $T$.

Пусть $\Gamma_{0}-$ подгрупша $\Gamma$, состоящая из всех элементов четной длины. Нетрудно проверить, что $\Gamma_{0}$ - это свободная группа ранга 2 с базисом, состоящим, например, из $x y$ и $y z$. Индекс $\left|\Gamma: \Gamma_{0}\right|$ равен 2 . Группа $\Gamma_{0}$ свободно действует на $T$. При этом фактор-граф $\Theta=\Gamma_{0} \backslash T$ имеет 2 вершины (соответствующие смежньм классам $\Gamma_{0}$ и $\left.\Gamma_{0} x\right)$, а также три ребра с метками $x, y, z$, которые соединяют эти две вершины.

Припишем метку "+" всем вершинам $T$, находящимся в одной орбите с $1 \in T$ относительно действия $\Gamma_{0}$. Всем оставшимся вершинам припишем метку “-”. В зависимости от знака вершины будем называть их положительными или отрицательными.

ОпРеДЕлЕниЕ. Размеченный граф $V$ назовем $\Theta$-графом, если выполнены следующие условия:

1) каждая вершина $v \in V$ имеет метку "+" или “-";

2) любое ребро $e \in V$ имеет метку $\mu(e)$, равную $x, y$ или $z$ и соединяет две вершины с разными метками;

3) из каждой вершины выходит не более одного ребра с данной меткой (следовательно, степень каждой вершины не более 3 ).

Отметим, что любая петля в $\Theta$-графе состоит из четного числа ребер по условию 2). Определим метку пути $p=e_{1} e_{2} \ldots e_{m}$ в $\Theta$-графе как произведение $\mu\left(e_{1}\right) \mu\left(e_{2}\right) \cdots \mu\left(e_{m}\right) \in \Gamma$. Из сказанного выше следует, что метка любой петли лежит в подг руппе $\Gamma_{0}$.

Примером $\Theta$-графа служит размеченное дерево $T$. Вообше, $\Theta$-деревом мы будем называть связный односвязный $\Theta$-граф̆. Поскольку любая подгруппа $H \subseteq \Gamma_{0}$

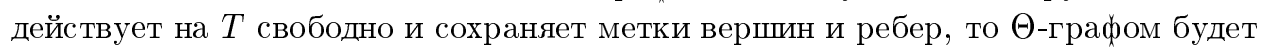
любой фактор-граф $H \backslash T$, в частности, и граф $\Theta$. 
Морфизмом $\varphi: A \rightarrow B$ одного $\Theta$-графа в другой назовем отображение, переводяшее вершины $A$ в вершины $B$, ребра $A$ в ребра $B$, с сохранением всех меток и отношения инцидентности. Например, для любой подгрупшы $H \subseteq \Gamma_{0}$ каноническая проекция $p_{H}: T \rightarrow H \backslash T$ будет морфизмом. $\Theta$-графы и их морфизмы образуют категорию, которую мы обозначим $\Theta$-Graphs. Нетрудно видеть, что $\Theta$ будет финальным объектом данной категории.

В категории $\Theta$-Graphs сушествуют суммы (дизъюнктные объединения) и прямые произведения объектов. Дадим явную конструкцию произведения $C=A \times B$ двух $\Theta$-графов. Для любого $\Theta$-графа $A$ обозначим $A_{+}^{0}\left(A_{-}^{0}\right)$ множество его положительных (отрицательных) вершин. Положим $C_{+}^{0}=A_{+}^{0} \times B_{+}^{0}$ и $C_{-}^{0}=A_{-}^{0} \times B_{-}^{0}$. Ребро с меткой $x(y, z)$ соединяет вершину $c_{1}=\left(a_{1}, b_{1}\right)$ с вершиной $c_{2}=\left(a_{2}, b_{2}\right)$ тогда и только тогда, когда два некоторых ребра с той же меткой соединяют вершины $a_{1}, a_{2}$ в $A$ и вершины $b_{1}, b_{2}$ в $B$, соответственно.

Сумма и произведение связаны между собой дистрибутивным законом

$$
X \times(Y \sqcup Z) \cong X \times Y \sqcup X \times Z .
$$

Пусть $V-\Theta$-граф. Назовем сопряжсенным графом граф $V^{*}$, получаемый из $V$ заменой меток всех вершин $V$ на противоположные. Очевидно, что $\left(V^{*}\right)^{*}=V$.

Если $A$ - конечный $\Theta$-граф, то обозначим $n_{3}(A)\left(n_{3}^{+}(A), n_{3}^{-}(A)\right)$ число всех его (соответственно, положительных, отрицательных) вершин степени 3. Заметим, что всегда $n_{3}^{+}(A)=n_{3}^{-}\left(A^{*}\right)$ и $n_{3}^{-}(A)=n_{3}^{+}\left(A^{*}\right)$, и, кроме того, $n_{3}(A)=n_{3}^{+}(A)+n_{3}^{-}(A)$.

Лемма 1.1. Пусть $A$ и $B$ - конечные $\Theta$-графьи. Тогда

1) $n_{3}^{+}(A \times B)=n_{3}^{+}(A) n_{3}^{+}(B)$;

2) $n_{3}^{-}(A \times B)=n_{3}^{-}(A) n_{3}^{-}(B)$;

3) $n_{3}\left(A \times\left(B \sqcup B^{*}\right)\right)=n_{3}(A) n_{3}(B)$.

ДокАЗАТЕЛЬСтво. Первые два утверждения вытекают из определения произведения $\Theta$-графов, если учесть то, что вершине степени 3 в произведении $A \times B$ соответствует пара вершин, каждая из которых имеет степень 3 . Второе утверждение получается из первого:

$$
\begin{aligned}
n_{3}\left(A \times\left(B \sqcup B^{*}\right)\right) & =n_{3}(A \times B)+n_{3}\left(A \times B^{*}\right) \\
& =n_{3}^{+}(A \times B)+n_{3}^{-}(A \times B)+n_{3}^{+}\left(A \times B^{*}\right)+n_{3}^{-}\left(A \times B^{*}\right) \\
& =n_{3}^{+}(A) n_{3}^{+}(B)+n_{3}^{-}(A) n_{3}^{-}(B)+n_{3}^{+}(A) n_{3}^{-}(B)+n_{3}^{-}(A) n_{3}^{+}(B) \\
& =\left(n_{3}^{+}(A)+n_{3}^{-}(A)\right)\left(n_{3}^{+}(B)+n_{3}^{-}(B)\right)=n_{3}(A) n_{3}(B) .
\end{aligned}
$$

Лемма доказана.

1.2. Эйлеровы характеристики графов и подгрупп. Напомним некоторые топологические результаты о графах. Пусть $X$ - некоторый связный граф, не являющийся деревом. Существует однозначно определенный подграф $c(X) \subseteq X$, называемый ядром $X$, которьй может быть охарактеризован следующими свойствами:

1) $c(X)$ - деформационный ретракт $X$; в частности, если $v \in c(X) \subseteq X-$ некоторая вершина, то $\pi_{1}(X, v) \cong \pi_{1}(c(X), v)$;

2) $c(X)$ - наименьший подграф графа $X$ со свойством 1$)$. 
Можно показать, что $c(X)$ представляет собой объединение всех минимальных нестягиваемых петель в графе $X$.

Отметим еше одно свойство $c(X)$. Очевидно, что в силу своей минимальности $c(X)$ не может содержать вершины степени 1 , так как иначе единственное ребро, инцидентное этой вершине, можно было бы стянуть к другому его концу.

Если $X$ является $\Theta$-графом, то ясно, что и его ядро $c(X)$ также является $\Theta$-графом (в случае дерева будем считать, что ядро пусто). Также можно считать, что

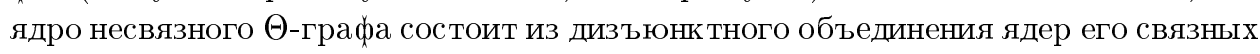

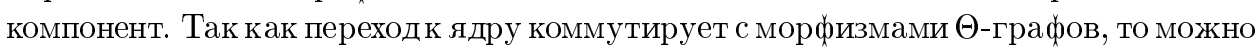
рассматривать его как функтор из категории $\Theta-$ Graphs в себя.

Пусть $H$ - нетривиальная подгруппа $\Gamma_{0}$. Положим $\Delta_{H}=H \backslash T$ (с отмеченной точкой $\{H\})$. Поскольку $\pi_{1}\left(\Delta_{H},\{H\}\right) \cong H$, то граф $\Delta_{H}$ не является деревом, и можно рассмотреть его ядро $c\left(\Delta_{H}\right)$. Степень любой вершины $\Delta_{H}$ равна 3 , следовательно, вершины $c\left(\Delta_{H}\right)$ имеют степень только 2 или 3 . Вершины степени 3 назовем точками ветвления. Докажем следующую лемму, характеризующую конечно порожденные подгруппы $H \subseteq \Gamma_{0}$.

Лемма 1.2. Нетривиальная подгруппа $H \subseteq \Gamma_{0}$ конечно порождена тогда и только тогда, когда $\Theta$-граф $c\left(\Delta_{H}\right)$ конечен.

ДоказАтельство. Выберем некоторую вершину $v \in c\left(\Delta_{H}\right)$ и конечный набор $l_{i}$ петель с началом и концом в $v$, который порождает $\pi_{1}\left(c\left(\Delta_{H}\right), v\right)$. Пусть $V \subseteq c\left(\Delta_{H}\right)$ - объединение всех этих петель. Ясно, что $v \in V$ и что $V$ - конечный связный граф. Поскольку набор $l_{i}$ порождает $\pi_{1}\left(c\left(\Delta_{H}\right), v\right)$, то можно считать, что любая петля $l$ с началом и концом в $v$, представляющая некоторый элемент из $\pi_{1}\left(c\left(\Delta_{H}\right), v\right)$, целиком лежит в $V$. Более того, любая минимальная нестягиваемая петля также лежит в $V$. Из того, что $c\left(\Delta_{H}\right)$ есть объединение всех таких петель, следует равенство $V=c\left(\Delta_{H}\right)$, т.е. граф $c\left(\Delta_{H}\right)$ конечен.

В обратную сторону утверждение очевидно, так как $H \cong \pi_{1}\left(c\left(\Delta_{H}\right), v\right)$.

СлЕДСТВИЕ 1.1. Пусть $r_{0}(H)$ обозначает $\operatorname{rank}(H)-1$. Тогда

$$
n_{3}\left(c\left(\Delta_{H}\right)\right)=2 r_{0}(H) .
$$

ДокАЗАТЕльство. Как известно, фундаментальная группа связного графа $V$ является свободной, и если граф $V$ конечен, то $\operatorname{rank}\left(\pi_{1}(V, v)\right)=1-\chi(V)$, где $\chi(V)$ - эйлерова характеристика графа $V$ (разность $\left|V^{0}\right|-\left|V^{1}\right|$ между числом вершин и числом ребер графа $V)$. Таким образом, $\operatorname{rank}(H)-1=-\chi\left(c\left(\Delta_{H}\right)\right)$.

Для произвольного конечного связного графа рассмотрим

$$
\sum_{v \in V^{0}} \operatorname{deg}(v)
$$

Поскольку любое ребро $e \in V^{1}$ дает вклад 2 в эту сумму, то

$$
\sum_{v \in V^{0}} \operatorname{deg}(v)=\sum_{e \in V^{1}} 2=2\left(\left|V^{1}\right|-\left|V^{0}\right|+\left|V^{0}\right|\right)=-2 \chi(V)+\sum_{v \in V^{0}} 2 .
$$


Отсюда получаем

$$
\sum_{v \in V^{0}}(\operatorname{deg}(v)-2)=-2 \chi(V) .
$$

Граф $c\left(\Delta_{H}\right)$ имеет вершины только степени 2 и 3 . Поэтому число точек ветвления $n_{3}\left(c\left(\Delta_{H}\right)\right)$ равно $-2 \chi\left(c\left(\Delta_{H}\right)\right)=2 r_{0}(H)$. Следствие доказано.

Рассмотрим теперь, что происходит с графом $\Delta_{H}$ при переходе от подгруппы $H \subseteq \Gamma_{0}$ к сопряженной подгруппе $a H a^{-1}, a \in \Gamma$.

ПРЕДЛОЖЕНИЕ 1. Пусть $H$ - нетривиальная подгруппа $\Gamma_{0}$ u $а \in \Gamma$. Тогда

1) $\Delta_{a a^{-1}} \cong \Delta_{H}($ изоморфизм $\Theta$-графов $)$, если $a \in \Gamma_{0}, u \Delta_{a H a^{-1}} \cong \Delta_{H}^{*}$, если $a \in \Gamma \backslash \Gamma_{0}$;

2) $c\left(\Delta_{a H a^{-1}}\right) \cong c\left(\Delta_{H}\right)$, ecли $a \in \Gamma_{0}, u c\left(\Delta_{a H a^{-1}}\right) \cong c\left(\Delta_{H}\right)^{*}$, ecли $a \in \Gamma \backslash \Gamma_{0}$.

ДокаЗАтельство. Пусть $p_{H}: T \rightarrow \Delta_{H}$ - каноническая проекция. Рассмотрим коммутативную диаграмму

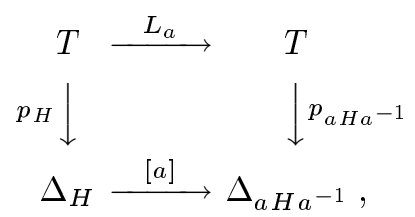

где $L_{a}$ - левый сдвиг на $a$, а отображение $[a]$ переводит вершину $H t$ в вершину $a H a^{-1}(a t)$, ребро $H e$ в ребро $a H a^{-1}(a e)$. Поскольку $L_{a^{-1}} L_{a}=L_{1}=\mathrm{Id}$, то $\left[a^{-1}\right]: \Delta_{a a^{-1}} \rightarrow \Delta_{H}$ будет обратным к $[a]$ отображением графов (но, вообше го-

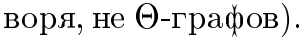

Пусть $a \in \Gamma_{0}$. Заметим, что $L_{a}: T \rightarrow T$ в этом случае является автоморфизмом $\Theta$-графа $T$ (сохраняет метки вершин), поэтому и индуцированное отображение $[a]$ также есть изоморфизм $\Theta$-графов. Если же $a \in \Gamma \backslash \Gamma_{0}$, то $L_{a}$ отображает любую положительную вершину $T$ в отрицательную и наоборот. Поскольку канонические проекции сохраняют метки, то [a] будет обрашать метки вершин и, следовательно, задавать изоморфизм $\Delta_{a H a^{-1}} \cong \Delta_{H}^{*}$. Второе утверждение непосредственно вытекает из первого.

ЗАмечаниЕ. Мы показали, что если нетривиальные подгруппы $H$ и $K$ сопряжены в $\Gamma_{0}$, то $\Theta$-графы $c\left(\Delta_{H}\right)$ и $c\left(\Delta_{K}\right)$ изоморфны. Верно и обратное: если $\Theta$-графы $c\left(\Delta_{H}\right)$ и $c\left(\Delta_{K}\right)$ изоморфны, то подгруппы $H$ и $K$ сопряжены в $\Gamma_{0}$. Действительно, выберем положительную вершину $v$ в $c\left(\Delta_{H}\right)$ (такая всегда сушествует). Группа $\pi_{1}\left(c\left(\Delta_{H}\right), v\right)$ может быть канонически вложена в $\Gamma_{0}$. Вложение $\mu: \pi_{1}\left(c\left(\Delta_{H}\right), v\right) \rightarrow \Gamma_{0}$ осушествляется следуюшим образом: пусть $l=e_{1} e_{2} \ldots e_{2 k}$ - петля, представляюшая элемент $g$ из $\pi_{1}\left(c\left(\Delta_{H}\right), v\right)$, где $e_{i}$ - последовательные ребра графа $c\left(\Delta_{H}\right)$. Положим $\mu(g)=\mu_{1}\left(e_{1}\right) \mu\left(e_{2}\right) \cdots \mu\left(e_{2 k}\right)$, где $\mu\left(e_{i}\right)$ - метка ребра $e_{i}$. Нетрудно показать, что если вершина $v$ соответствует орбите $H t$, то $\mu\left(\pi_{1}\left(c\left(\Delta_{H}\right), v\right)\right)=t^{-1} H t$. Поскольку $v$ - положительная вершина, то сопрягающий элемент $t$ должен быть из $\Gamma_{0}$. Итак, класс сопряженности подгруппы $H$ в $\Gamma_{0}$ определяется по $\Theta$-графу $c\left(\Delta_{H}\right)$ (точнее, по его классу изоморфизма) однозначно. На самом деле, любой связный $\Theta$-граф $\Delta$, не являющийся деревом, однозначно 
определяет класс сопряженности в $\Gamma_{0}$ некоторой подгруппы $H=H(c(\Delta))$ с помощь приведенной вьше конструкции.

Обратно, если подгруппа $H \subseteq \Gamma_{0}$ порождена элементами $h_{1}, \ldots, h_{m}$, то $\Theta$-граф $c\left(\Delta_{H}\right)$ строится эффективно за конечное число шагов. Алгоритм здесь таков. Надо взять букет из $m$ окружностей и разбить каждую из них на $\left|h_{i}\right|$ дуг, где $\left|h_{i}\right|$ означает длину $h_{i}$, т.е. расстояние от вершины 1 до вершины $h_{i}$ в $T$. Присвоим каждой дуге метку так, чтобы при обходе $i$-й окружности читалось бы слово $w_{i}$ в алфавите $S=\{x, y, z\}$, равное $h_{i}$ в группе $\Gamma$. Заметим, что каждая окружность разбита на четное число дуг. Начальной вершине букета присвоим метку “+”. Остальньм вершинам метки присваиваем так, чтобы плюсы и минусы чередовались. Затем последовательно склеиваем различные ребра с одинаковыми метками, выходящие из одной вершины (при этом каждый раз число ребер уменьшается на единицу). Последний шаг заключается в “отламывании” ребер, инцидентных вершинам степени 1.

Детали рассуждения мы оставляем читателю.

В дальнейшем будем обозначать через $H^{g}$ подгруппу $g \mathrm{Hg}^{-1}$, сопряженную к $H \subseteq \Gamma_{0}$ при помощи элемента $g \in \Gamma$.

ПРЕДЛОЖЕНИЕ 2. Пусть $H$ и $K$ - некоторые подгрупnы в $\Gamma_{0}$. Тогда

$$
\bigsqcup_{g \in H \backslash \Gamma_{0} / K} \Delta_{H \cap K g} \cong \Delta_{H} \times \Delta_{K}
$$

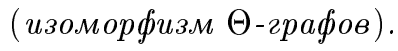

ДокАЗАТЕЛЬСТво. Изоморфизм

$$
f: \bigsqcup_{g \in H \backslash \Gamma_{0} / K} \Delta_{H \cap K^{g}} \rightarrow \Delta_{H} \times \Delta_{K}
$$

строится следующим образом. Зафиксируем некотороемножество представителей двойных смежных классов $g \in H \backslash \Gamma_{0} / K$. Положим

$$
\begin{aligned}
& f\left(\left(H \cap K^{g}\right) t\right)=\left(H t, K g^{-1} t\right), \quad t \in T^{0} \\
& f\left(\left(H \cap K^{g}\right) e\right)=\left(H e, K g^{-1} e\right), \quad e \in T^{1} .
\end{aligned}
$$

Корректность $f$ проверяется непосредственно. Кроме того, так как $g \in \Gamma_{0}$, то $f$ отображает положительные вершины в положительные, а отрицательные в отрицательные. Легко видеть, что $f$ сохраняет метки ребер и отношение инцидент-

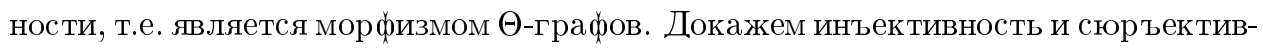
ность $f$.

Инъективность $f$. Пусть $\left(H t, K g_{1}^{-1} t\right)=\left(H s, K g_{2}^{-1} s\right), t, s \in T^{0}$. Отсюда $H t=H s, K g_{1}^{-1} t=K g_{2}^{-1} s$ и можно записать $t=h s=g_{1} k g_{2}^{-1} s$ для некоторых элементов $h \in H, k \in K$. Поскольку $\Gamma_{0}$ действует на вершинах дерева $T$ одного знака свободно транзитивно, получаем, что $h=g_{1} k g_{2}^{-1}$, т.е. $H g_{2} K=H g_{1} K$. Поэтому $g_{1}=g_{2}=g$ и $h=g \mathrm{~kg}^{-1} \in H \cap K^{g}$. Инъективность для вершин доказана.

Сюръективность $f$. Пусть $(H t, K s) \in \Delta_{H} \times \Delta_{K}-$ некоторая вершина. Надо найти такие $t^{\prime} \in T$ и некоторьй двойной смежный класс $H \backslash g / K$, что $H t^{\prime}=H t$, 
$K g^{-1} t^{\prime}=K s$. Отсюда $t^{\prime}=h t=g k s$ для некоторых $h \in H$ и $k \in K$. Вершины $t$ и $s$ можно рассматривать как элементы $\Gamma$, причем $t s^{-1} \in \Gamma_{0}(t$ и $s$ как вершины должны быть одного знака). Из равенства $h t=g k s$ вытекает, что $H t s^{-1} K=H g K$ и, значит, $g$ как представитель двойного смежного класса определяется однозначно. Из последнего равенства вытекает также, что $t s^{-1}=h^{-1} g k$ для некоторых $h$ и $k$. Положим $t^{\prime}=h t=g k s$. Это и будет нужная вершина дерева $T$.

Доказательство биективности отображения $f$ для ребер $T$ проводится совершенно аналогично. Предложение доказано.

ПРЕДЛОЖЕНИЕ 3. Пусть $Н$ и К две нетривиальнье подгруппы $\Gamma_{0}$. Имеет место следующая коммутативная диаграмма в категории $\Theta$-Graphs

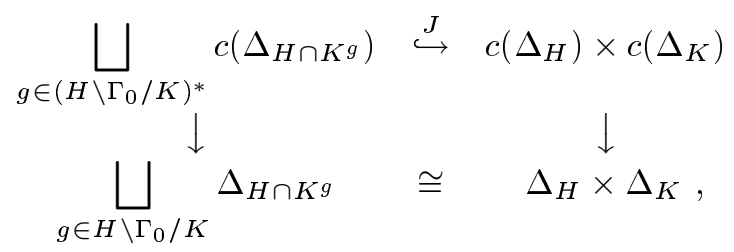

в которой вертикальнье стрелки обозначают естественнье вложсения графов.

Здесь и далее в работе “*” при суммировании по двойным смежным классам означает, что берутся только те классы $H g K$, для которых группа $H \cap K^{g}$ бесконечна.

В доказательстве нуждается только утверждение о верхнем вложении $J$. Рассмотрим нетривиальную подгрупу $H \cap K^{g}$ (если такая существует). Пусть имеется минимальная нестягиваемая петля $l=e_{1} e_{2} \ldots e_{2 n}$ в $c\left(\Delta_{H \cap K^{g}}\right)$. Поскольку при отображении $f$, построенном при доказательстве предложения 2 , петля $l$ переходит в произведение петель $l_{1}$ и $l_{2}$, каждая из которых имеет ту же самую метку $\mu\left(e_{1}\right) \mu\left(e_{2}\right) \cdots \mu\left(e_{2 n}\right)$, что и $l$, то петли $l_{1}$ и $l_{2}$ будут также минимальными нестягиваемыми петлями в соответствуюших графах $c\left(\Delta_{H}\right)$ и $c\left(\Delta_{K}\right)$. По свойствам ядер графов отсюда вытекает, что $l_{1}$ и $l_{2}$ целиком лежат в $c\left(\Delta_{H}\right)$ и $c\left(\Delta_{K}\right)$. Так как $c\left(\Delta_{H \cap K^{g}}\right)$ есть не что иное, как объединение всех минимальных нестягивамых петель в $\Delta_{H \cap K^{g}}$, то предложение можно считать доказанным.

СЛЕДСТВИЕ 1.2. Если в условиях предложения 3 подгруппы $H$ и $K$ конечно порождены, то существует только конечное число двойных смежных классов $H g K$ таких, что подгруппа $H \cap K^{g}$ нетривиальна. При этом она будет конечно порожденной.

Действительно, рассмотрим вложение $J$. Графы $c\left(\Delta_{H}\right)$ и $c\left(\Delta_{K}\right)$ конечны по лемме 1.2. Так как $c\left(\Delta_{H \cap K^{g}}\right)$ не пусто, если $H \cap K^{g}$-нетривиальная подгруппа, то число таких двойных смежных классов $H g K$ будет конечньм. Ясно к тому же, что граф̆ $c\left(\Delta_{H \cap K^{g}}\right)$ должен быть в этом случае конечным, поэтому по той же лемме 1.2 подгруппа $H \cap K^{g}$ конечно порождена.

ПРЕДЛОЖЕНИЕ 4. Пусть Н и $\mathrm{K}$ - две нетривиальные конечно порожденнье подгруппь $\Gamma_{0}$ и пусть $K^{x}-$ подгруппа, сопряженная с $K$ при помощи 
одной из образующих $x \in \Gamma$. Тогда

$$
\sum_{g \in\left(H \backslash \Gamma_{0} / K\right)^{*}} r_{0}\left(H \cap K^{g}\right)+\sum_{g \in\left(H \backslash \Gamma_{0} / K^{x}\right)^{*}} r_{0}\left(H \cap\left(K^{x}\right)^{g}\right) \leqslant 2 r_{0}(H) r_{0}(K) .
$$

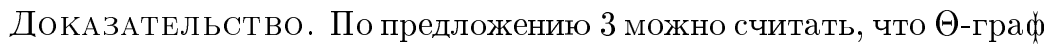

$$
\bigsqcup_{g \in\left(H \backslash \Gamma_{0} / K\right)^{*}} c\left(\Delta_{H \cap K^{g}}\right) \sqcup \bigsqcup_{g \in\left(H \backslash \Gamma_{0} / K^{x}\right)^{*}} c\left(\Delta_{H \cap\left(K^{x}\right)^{g}}\right)
$$

вложен в произведение

$$
c\left(\Delta_{H}\right) \times\left(c\left(\Delta_{K}\right) \sqcup c\left(\Delta_{x K x^{-1}}\right)\right) \cong c\left(\Delta_{H}\right) \times\left(c\left(\Delta_{K}\right) \sqcup c\left(\Delta_{K}\right)^{*}\right) .
$$

Сравним число точек ветвления в обоих графах

$$
\begin{aligned}
\sum_{g \in\left(H \backslash \Gamma_{0} / K\right)^{*}} n_{3}\left(c\left(\Delta_{H \cap K^{g}}\right)\right)+\sum_{g \in\left(H \backslash \Gamma_{0} / K^{x}\right)^{*}} n_{3}\left(c\left(\Delta_{H \cap\left(K^{x}\right)^{g}}\right)\right) & \\
& \leqslant n_{3}\left(c\left(\Delta_{H}\right) \times\left(c\left(\Delta_{K}\right) \sqcup c\left(\Delta_{K}\right)^{*}\right)\right) .
\end{aligned}
$$

По следствию к лемме 1.2 левая часть неравенства равна

$$
\sum_{g \in\left(H \backslash \Gamma_{0} / K\right)^{*}} 2 r_{0}\left(H \cap K^{g}\right)+\sum_{g \in\left(H \backslash \Gamma_{0} / K^{x}\right)^{*}} 2 r_{0}\left(H \cap\left(K^{x}\right)^{g}\right) .
$$

По этому же следствию и лемме 1.1 правая часть равна $4 r_{0}(H) r_{0}(K)$. Сократив на 2 , получаем нужное утверждение. Предложение доказано.

\section{§ 2. Доказательство гипотезы Х. Нейман}

2.1. ОпреДЕлЕнИЕ. Скажем, что группа $G$ имеет тип NVF, если она является неэлементарной (не почти циклической) конечно порожденной почти свободной группой, т.е. группой, содержащей некоторую нециклическую конечно порожденную свободную подгруппу $G_{0}$ конечного индекса.

Вообше говоря, группы типа NVF можно рассматривать как объекты соответствуюшей категории, морфизмами которой являются вложения групा, но этот факт здесь не будет использован.

Как известно, любая группа $G$ типа NVF обладает рациональной эйлеровой характеристикой $\chi(G)$, которая равна $\left(1-\operatorname{rank}\left(G_{0}\right)\right) /\left|G: G_{0}\right|$, где $G_{0} \subseteq G$ - свободная подгруппа конечного индекса $\left|G: G_{0}\right|$. Заметим, что для всех групп типа NVF эйлерова характеристика строго отрицательна. Для определенных в предыдущем разделе групп $Г$ и $\Gamma_{0}$ эйлерова характеристика принимает значение $\chi(\Gamma)=-\frac{1}{2}$ и $\chi\left(\Gamma_{0}\right)=-1$.

Возьмем некоторую группу $G$ типа NVF. Напомним конструкцию коммутативного ассоциативного кольца с единицей $D(G)$, ассоциированного с группой $G$, которая подробно описана в [1] для класса бесконечных гиперболических (в смысле Громова) групп, и, в частности, для групп типа NVF. 
Как $\mathbb{Z}$-модуль $D(G)$ состоит из конечных сумм вида $\sum n_{i}\left[H_{i}\right]$, где все $n_{i}$ - целые числа, а все $\left[H_{i}\right]$ - классы сопряженности в группе $G$ бесконечных конечно порожденных подгрупп $H_{i}$, т.е. классы сопряженности таких подгрупп составляют аддитивный базис кольца $D(G)$. Произведение двух базисных элементов определяется по следующей формуле (подгруппы $H$ и $K$, представляюшие соответствуюшие классы сопряженности, считаются фиксированньми)

$$
[H][K]=\sum_{g \in(H \backslash G / K)^{*}}\left[H \cap K^{g}\right] .
$$

Произведение по аддитивности можно распространить на $D(G)$.

ЗАмЕчАниЕ 1. В [1] показано, что это умножение корректно определено, т.е. не зависит от выбора представителей классов сопряженности. Кроме того, сумма всегда будет конечной и состоять из классов сопряженности бесконечных конечно порожденных подгрупп группы $G$. Это умножение обладает свойствами ассоциативности и коммутативности. Единицей кольца $D(G)$ служит класс $[G]$.

Замечание 2. Умножение в кольце $D\left(\Gamma_{0}\right)$ прямо связано с умножением $\Theta$-графов. Рассмотрим две бесконечные конечно порожденные подгрупшы $H, K$ в $\Gamma_{0}$ и соответствуюшие им графы $\Delta_{H}, \Delta_{K}$ (зависящие только от классов сопряженности $[H],[K])$, а также их ядра $c\left(\Delta_{H}\right)$ и $c\left(\Delta_{K}\right)$, которые будут конечньми по лемме 1.2. Пусть $\Delta_{1}, \ldots, \Delta_{m}$ - все связные компоненты произведения $c\left(\Delta_{H}\right) \times c\left(\Delta_{K}\right)$, не являюшиеся деревьями. $\Theta$-графам $c\left(\Delta_{1}\right), \ldots, c\left(\Delta_{m}\right)$ однозначно соответствуют классы сопряженности $\left[H_{1}\right], \ldots,\left[H_{m}\right]$ некоторых конечно порожденных подгрупп в $\Gamma_{0}$ (см. замечание после предложения 1$)$. Сумма всех этих классов сопряженности в кольце $D\left(\Gamma_{0}\right)$ равна произведению $[H][K]$.

$\mathrm{C}$ другой стороны, можно показать, что кольцо $D\left(\Gamma_{0}\right)$ (и аналогичные кольца $D(G))$ является группой Гротендика $K_{0}$ некоторой категории, а именно, категории конечных $\Theta$-графов, совпадающих со своими ядрами.

Каждая конечно порожденная подгруппа $H$ группы $G$ будет почти свободной группой с эйлеровой характеристикой

$$
\chi(H)=\chi\left(H \cap G_{0}\right) /\left|H: H \cap G_{0}\right|
$$

(очевидно, что индекс $\left|H: H \cap G_{0}\right|$ конечен). Поскольку на классе сопряженности подгруппы $H$ значение эйлеровой характеристики постоянно, то можно определить рациональное число $\chi([H])$ для всего класса сопряженности подгруппы $H$. Заметим, что для бесконечной конечно порожденной подгруппы $H$ число $\chi(H)$ равно нулю в том и только том случае, когда $H$ - почти циклическая подгруппа, в противном случае, $\chi(H)<0$.

Продолжим $\chi$ по аддитивности до гомоморфизма абелевых групп $\chi: D(G) \rightarrow \mathbb{Q}$ $(\mathbb{Q}$ - поле рациональных чисел), который мы также будем называть әйлеровой $x a$ рактеристикой. Приведенной әйлеровой характеристикой будем называть гомоморфизм абелевых групп $r: D(G) \rightarrow \mathbb{Q}$, отличающийся от эйлеровой характеристики лишш нормируюшим множителем $1 / \chi([G])$ (таким образом, $r([H]$ ) равно $\chi(H) / \chi(G))$. В частности, если $G$ - свободная группа ранга 2 , а $H$ - ее нетривиальная (бесконечная) конечно порожденная подгруппа, то

$$
r([H])=-\chi([H])=\operatorname{rank}(H)-1=r_{0}(H) .
$$


ОБОБШЕННАЯ ГИПОТЕЗА Х. НЕЙМАН. Пусть $G$ - гpynna muna NVF, $G_{0}-e e$ свободная подгруппа (минимального) конечного индекса, $и[H],[K] \in D(G)$. Тогда выполнено следующее неравенство (неравенство Х. Нейман)

$$
r([H][K]) \leqslant\left|\chi\left(G_{0}\right)\right| r([H]) r([K]) .
$$

В более подробной записи

$$
\sum_{g \in(H \backslash G / K)^{*}} r\left(\left[H \cap K^{g}\right]\right) \leqslant\left|\chi\left(G_{0}\right)\right| r([H]) r([K]) .
$$

Связь этой гипотезы с классической гипотезой Х. Нейман об оценке ранга пересечения двух нетривиальных конечно порожденных подгрупп $H, K$ свободной группы $F$ и ее более сильным вариантом, предложенным У. Нейманом, уже отмечалась во введении.

Будем говорить, что для группы $G$ типа NVF выполнено неравенство Х. Нейман, если неравенство (2.1) выполнено в кольце $D(G)$.

Основным результатом работы является

Теорема 1. Для группь $\Gamma=(\mathbb{Z} / 2 \mathbb{Z}) *(\mathbb{Z} / 2 \mathbb{Z}) *(\mathbb{Z} / 2 \mathbb{Z})$ выполнено неравенство Х. Нейман

$$
r([H][K]) \leqslant r([H]) r([K]) .
$$

ДокАЗАТЕЛЬСТво будет проведено в два шага.

Шаг 1. Будем работать пока с произвольной группой $G$ типа NVF. Выберем подгруппы $H$ и $K$, представляющие классы сопряженности $[H],[K]$. Покажем, как свести доказательство неравенства (2.1) к доказательству того же неравенства для $[L]$ и $[P]$, где $L, P$ - некоторые подгруппы конечного индекса в $H$ и $K$, соответственно.

Лемма 2.1. Пусть $G$ - группа типа NVF; $H$ и $K$ - две ее бесконечные конечно порохденные подгруппы и $L$ - подгруппа конечного индекса в $H$. Тогда

$$
\sum_{\gamma \in(L \backslash G / K)^{*}} \chi\left(L \cap K^{\gamma}\right)=|H: L| \sum_{g \in(H \backslash G / K)^{*}} \chi\left(H \cap K^{g}\right) .
$$

ДокАЗАТЕльство. Зафиксируем представители $g$ множества двойных смежных классов $H \backslash G / K$. Для данного $g$ положим $K_{g}=H \cap K^{g} \subseteq H$. Пусть $T(g)$ - множество представителей двойных смежных классов $L \backslash H / K_{g}$ в подгруппе $H$. Так как индекс подгруппы $L$ в $H$ конечен, то $T(g)$ - конечное множество для любого $g$.

Непосредственной проверкой убеждаемся, что произвольный двойной смежньй класс $H g K$ представляется в следуюшем виде:

$$
H g K=\bigsqcup_{h \in T(g)} L h g K .
$$

Пусть $h \in T(g)$. Рассмотрим подгруппу

$$
L \cap K^{h g}=L \cap h g K(h g)^{-1}=h\left(h^{-1} L h \cap K^{g}\right) h^{-1} .
$$


Ю. C. CEMEHOB

Индекс $\left|H: h^{-1} L h\right|$ конечен для любого $h \in H$. Поскольку

$$
h^{-1} L h \cap K^{g}=H \cap h^{-1} L h \cap K^{g}=h^{-1} L h \cap H \cap K^{g}=h^{-1} L h \cap K_{g}
$$

и $K_{g} \subseteq H$, то индекс $\left|K_{g}: h^{-1} L h \cap K_{g}\right|$ также конечен. Следовательно, подгруппа $L \cap K^{h g} \cong h^{-1} L h \cap K_{g}$ бесконечна тогда и только тогда, когда бесконечна подгруппа $K_{g}$. Отсюда вытекает равенство

$$
\sum_{\gamma \in(L \backslash G / K)^{*}} \chi\left(L \cap K^{\gamma}\right)=\sum_{g \in(H \backslash G / K)^{*}} \sum_{h \in T(g)} \chi\left(h^{-1} L h \cap K_{g}\right) .
$$

Так как индекс $\left|K_{g}: K_{g} \cap h^{-1} L h\right|<\infty$, то по известной формуле для эйлеровых характеристик

$$
\chi\left(h^{-1} L h \cap K_{g}\right)=\left|K_{g}: K_{g} \cap h^{-1} L h\right| \chi\left(K_{g}\right)=\left|K_{g}: K_{g} \cap h^{-1} L h\right| \chi\left(H \cap K^{g}\right) .
$$

Подсчитаем сумму

$$
\sum_{h \in T(g)}\left|K_{g}: K_{g} \cap h^{-1} L h\right|
$$

Для этого рассмотрим правое действие подгруппы $K_{g} \subseteq H$ на конечном множестве правых смежных классов $L \backslash H$

$$
R_{k}: L h \rightarrow L\left(h k^{-1}\right), \quad k \in K_{g} .
$$

Стабилизатор точки $L h$ есть подгруппа $K_{g} \cap h^{-1} L h$. Точки $L h_{1}$ и $L h_{2}$ принадлежат одной $K_{g}$-орбите в том и только том случае, когда $L h_{1} K_{g}=L h_{1} K_{g}$, т.е. множество $T(g)$ параметризует орбиты этого действия. Поэтому сумма (2.4) есть в точности число точек в множестве $L \backslash H$, равное индексу $|H: L|$. Итак,

$$
\sum_{g \in(H \backslash G / K)^{*}} \sum_{h \in T(g)} \chi\left(h^{-1} L h \cap K_{g}\right)=\sum_{g \in(H \backslash G / K)^{*}}|H: L| \chi\left(H \cap K^{g}\right) .
$$

Лемма доказана.

СлЕДСтвиЕ. Справедливо следующее равенство $r([L][K])=|H: L| r([H][K])$.

Непосредственно получается из (2.3) умножением на $1 / \chi(G)$.

Пусть $L \subseteq H$ и $P \subseteq K$ - подгруппы конечного индекса в $H$ и $K$ такие, что вьполнено неравенство $r([L][P]) \leqslant\left|\chi\left(G_{0}\right)\right| r([L]) r([P])$. Поскольку умножение в кольце $D(G)$ коммутативно, то по предыдущему следствию

$$
r([L][P])=|H: L||K: P| r([H][K]) .
$$

Очевидно, что справедливо равенство

$$
r([L]) r([P])=|H: L||K: P| r([H]) r([K]) .
$$

Таким образом, неравенство $r([H][K]) \leqslant\left|\chi\left(G_{0}\right)\right| r([H]) r([K])$ также выполняется. 
Шаг 2. Предыдущие рассуждения показывают, что в группе Г можно доказывать неравенство Х. Нейман для классов сопряженности подгрупп $L=H \cap \Gamma_{0}$ и $P=K \cap \Gamma_{0}$. Поскольку $\Gamma_{0}-$ свободная группа ранга 2 , то подгруппы $L, P$ будут бесконечньми конечно порожденньми свободными группами.

Необходимо показать, что

$$
\sum_{g \in(L \backslash \Gamma / P)^{*}} r\left(\left[L \cap P^{g}\right]\right) \leqslant r([L]) r([P]) .
$$

Поскольку для $\Gamma=(\mathbb{Z} / 2 \mathbb{Z}) *(\mathbb{Z} / 2 \mathbb{Z}) *(\mathbb{Z} / 2 \mathbb{Z})$ эйлерова характеристика равна $-\frac{1}{2}$, то для любой бесконечной конечно порожденной свободной подгруппы $W \subset \Gamma$

$$
r([W])=\chi(W) / \chi(\Gamma)=2(\operatorname{rank}(W)-1)=2 r_{0}(W) .
$$

Таким образом, надо показать, что

$$
\sum_{g \in(L \backslash \Gamma / P)^{*}} r_{0}\left(L \cap P^{g}\right) \leqslant 2 r_{0}(L) r_{0}(P) .
$$

Рассмотрим левую часть этого неравенства. Обозначим через $U$ подмножество представителей $g$ двойных смежных классов $L \backslash \Gamma / K$ таких, что $L \cap P^{g}$ - бесконечная подгруппа (т.е. $\left.g \in(L \backslash \Gamma / P)^{*} \Leftrightarrow g \in U\right)$. Положим

$$
U_{0}=U \cap \Gamma_{0}, \quad U_{1}=U \cap \Gamma_{0} x=U \backslash U_{0}
$$

$(x-$ один из порождающих $\Gamma)$.

ЛЕмма 2.2. (i) $U_{0}$ - множество представителей $g$ всех двойных смежных классов $L \backslash \Gamma_{0} / P$ в группе $\Gamma_{0}$ таких, что подгруппа $L \cap P^{g}$ бесконечна.

(ii) $U_{1} x^{-1} \subset \Gamma_{0}$ - множество представителей $g$ всех двойных смежных классов $L \backslash \Gamma_{0} / P^{x}$ в группе $\Gamma_{0}$ таких, что подгруппа $L \cap\left(P^{x}\right)^{g}$ бесконечна.

ДокаЗАтЕльство. Утверждение (i) очевидно. Докажем утверждение (ii). Поскольку $U_{1} \subset \Gamma_{0} x$, то $U_{1} x^{-1} \subset \Gamma_{0}$. Предположим, что $L u_{1} x^{-1} P^{x}=L u_{2} x^{-1} P^{x}$. Отсюда следует, что двойные смежные классы $L u_{1} P$ и $L u_{2} P$ совпадают, т.е. $u_{1}=u_{2}$.

Пусть $g \in U_{1}$. Тогда

$$
L \cap g x^{-1} P^{x} x g^{-1}=L \cap P^{g} .
$$

Получаем, что $g x^{-1} \in\left(L \backslash \Gamma_{0} / P\right)^{*}$ тогда и только тогда, когда $g \in U_{1}$. Лемма доказана.

По этой лемме

$$
\sum_{g \in(L \backslash \Gamma / P)^{*}} r_{0}\left(L \cap P^{g}\right)=\sum_{g \in\left(L \backslash \Gamma_{0} / P\right)^{*}} r_{0}\left(L \cap P^{g}\right)+\sum_{g \in\left(L \backslash \Gamma_{0} / P^{x}\right)^{*}} r_{0}\left(L \cap\left(P^{x}\right)^{g}\right) .
$$

Нужная оценка теперь вытекает из неравенства (1.1), доказанного в предложении 4. Теорема доказана.

Назовем элемент $d=\sum n_{i}\left[H_{i}\right]$ кольца $D(G)$ неотрицательным (неположительныц $)$, если все $n_{i} \geqslant 0\left(n_{i} \leqslant 0\right)$. Обозначим множество неотрицательных (неположительных) элементов $D(G)_{+}\left(D(G)_{-}\right)$. 
СлеДствИЕ. Пусть $d_{1}, d_{2} \in D(\Gamma)_{+}-$два неотрицательных әлемента. Тог$\partial a r\left(d_{1} d_{2}\right) \leqslant r\left(d_{1}\right) r\left(d_{2}\right)$.

Доказательство очевидно.

Теорема 1 позволяет получить более общий результат.

Tеорема 2. Пусть группа $G$ типа NVF содержит свободное произведение $\Gamma=(\mathbb{Z} / 2 \mathbb{Z}) *(\mathbb{Z} / 2 \mathbb{Z}) *(\mathbb{Z} / 2 \mathbb{Z})$ в качестве подгруппьи конечного индекса. Тогда для группы $G$ выполнено неравенство Х. Нейман $\left(c\left|\chi\left(G_{0}\right)\right|=1\right)$.

ДокАЗАТЕЛЬство. Будем считать, что имеется вложение $j: \Gamma \rightarrow G$, и отождествим группу $\Gamma$ с ее образом при этом вложении. Классы сопряженности подгрупп в группе $G$ и группе $Г$ будем обозначать, соответственно, $[-]_{G}$ и $[-]_{\Gamma}$, а приведенные эйлеровы характеристики $-r_{G}$ и $r_{\Gamma}$.

В работе [1] показано, что гомоморфизм абелевых групп $\pi: D(G) \rightarrow D(\Gamma)$ такой, что

$$
\pi\left([H]_{G}\right)=\sum_{g \in(\Gamma \backslash G / H)^{*}}\left[\Gamma \cap H^{g}\right]_{\Gamma} \quad \forall[H]_{G} \in D(G),
$$

является в действительности гомоморфизмом колец (отметим, что множество $\Gamma \backslash G / H=(\Gamma \backslash G / H)^{*}$ конечно, поскольку $Г$ имеет конечный индекс в $\left.G\right)$.

Докажем равенство

$$
r_{\Gamma}\left(\pi\left([H]_{G}\right)\right)=r_{G}\left([H]_{G}\right) .
$$

Имеем

$$
r_{\Gamma}\left(\pi\left([H]_{G}\right)=\chi(\Gamma)^{-1} \sum_{g \in \Gamma \backslash G / H} \chi\left(\Gamma \cap H^{g}\right)=\chi(\Gamma)^{-1} \sum_{g \in \Gamma \backslash G / H} \chi\left(g^{-1} \Gamma g \cap H\right) .\right.
$$

Подгруппа $g^{-1} \Gamma g \cap H$ имеет конечный индекс в $H$. Рассматривая правое действие подгруппы $H$ на множестве правых смежных классов $\Gamma \backslash G$ (см. доказательство леммы 2.1.), получаем, что

$$
\sum_{g \in \Gamma \backslash G / H} \chi\left(g^{-1} \Gamma g \cap H\right)=\sum_{g \in \Gamma \backslash G / H}\left|H: g^{-1} \Gamma g \cap H\right| \chi(H)=|G: \Gamma| \chi(H) .
$$

Следовательно,

$$
r_{\Gamma}\left(\pi\left([H]_{G}\right)\right)=|G: \Gamma| \chi(H) / \chi(\Gamma)=\chi(H) / \chi(G)=r_{G}\left([H]_{G}\right) .
$$

Пусть теперь $[H]_{G},[K]_{G} \in D(G)$. Воспользуемся гомоморфизмом $\pi$.

$$
r_{G}\left([H]_{G} \cdot[K]_{G}\right)=r_{\Gamma}\left(\pi\left([H]_{G} \cdot[K]_{G}\right)\right)=r_{\Gamma}\left(\pi\left([H]_{G}\right) \cdot \pi\left([K]_{G}\right)\right) .
$$

Поскольку $\pi\left([H]_{G}\right)$ и $\pi\left([K]_{G}\right)$ принадлежат $D(\Gamma)_{+}$, то, по следствию к лемме 2.2 и равенству $(2.5)$,

$$
r_{\Gamma}\left(\pi\left([H]_{G}\right) \cdot \pi\left([K]_{G}\right)\right) \leqslant r_{\Gamma}\left(\pi\left([H]_{G}\right)\right) r_{\Gamma}\left(\pi\left([K]_{G}\right)\right)=r_{G}\left([H]_{G}\right) r_{G}\left([K]_{G}\right) .
$$

Теорема доказана. 
СлЕДСТВИЕ. Если для группы $H$ типа NVF выполнено неравенство Х. Нейман, то это неравенство выполнено и для любой группь $G$, в которую группа H вложена как подгруппа конечного индекса.

2.2. Конструкция банаховой алгебры $\widehat{A}(G)$. В этом разделе группа $G$ предполагается группой типа NVF, для которой выполнены условия теоремы 2. Рассмотрим кольцо $D(G)$. В этом кольце имеется идеал $E(G)$, состоящий из всевозможных конечных сумм вида $\sum n_{i}\left[E_{i}\right]$, в которых все подгруппы $E_{i}-$ бесконечные почти циклические подгруппы в $G$. Утверждение, что $E(G)$ - идеал, легко вытекает из формулы умножения в $D(G)$. Фактор-кольцо $D(G) / E(G)$ изоморфно кольцу $D N(G)$, определенному в [1]. Кольцо $D N(G)$ как $\mathbb{Z}$-модуль свободно порождается всеми классами сопряженности $[H]$ неэлементарных конечно порожденных подгрупп $H \subseteq G$. Произведение двух классов определяется по формуле

$$
[H][K]=\sum_{g \in(\Gamma \backslash G / H)^{* *}}\left[H \cap K^{g}\right],
$$

в которой “**” означает, что берутся только те двойные смежные классы $\mathrm{HgK}$, для которых группа $H \cap K^{g}$ неэлементарна.

На идеале $E(G)$ функции $\chi$ и $r$ тождественно равны 0 . С другой стороны, $\chi$ и $r$ очевидным образом определяются на кольце $D N(G)$. Заметим, что $r([H])>0$ для любого $[H] \in D N(G)$.

Пусть $A(G)=\mathbb{C} \otimes D N(G)$, т.е. $\mathbb{C}$-алгебра $A(G)$ состоит из конечных сумм вида $\sum c_{i}\left[H_{i}\right], c_{i} \in \mathbb{C}$.

Для любого $a=\sum c_{i}\left[H_{i}\right] \in A(G)$ положим $\|a\|=\sum\left|c_{i}\right| r\left(\left[H_{i}\right]\right)$.

ПРЕДЛОЖЕНИЕ 5. Норма $\|\cdot\|$ является мультипликативной на алгебpe $A(G)$.

ДоКАЗАТЕЛЬСТВо. Ясно, что $\|a\|=0$ тогда и только тогда, когда $a=0$ и $\|\lambda a\|=|\lambda|\|a\|$ для любых $a \in A(G)$ и $\lambda \in \mathbb{C}$. Неравенство $\|a+b\| \leqslant\|a\|+\|b\|$ также легко проверяется непосредственно. Остается проверить, что норма мультипликативна. Докажем сначала, что $\|[H][K]\| \leqslant\|[H]\| \times\|[K]\|$ для любых $[H],[K] \in A(G)$.

Действительно,

$$
\|[H][K]\|=\sum_{g \in(\Gamma \backslash G / H)^{* *}} r\left(\left[H \cap K^{g}\right]\right) \leqslant r([H]) r([K])=\|[H]\| \cdot\|[K]\|
$$

по неравенству Х. Нейман.

Пусть теперь $a=\sum_{i} \alpha_{i}\left[H_{i}\right]$ и $b=\sum_{j} \beta_{j}\left[K_{j}\right]$ лежат в $A(G)$. Тогда

$$
a \cdot b=\sum_{i, j} \alpha_{i} \beta_{j}\left[H_{i}\right]\left[K_{j}\right] .
$$

Из уже доказанных свойств нормы вытекает

$$
\|a \cdot b\| \leqslant \sum_{i, j}\left|\alpha_{i}\right| \cdot\left|\beta_{j}\right| \cdot\left\|\left[H_{i}\right]\left[K_{j}\right]\right\| \leqslant \sum_{i, j}\left|\alpha_{i}\right| \cdot\left|\beta_{j}\right| \cdot\left\|\left[H_{i}\right]\right\| \cdot\left\|\left[K_{j}\right]\right\|=\|a\| \cdot\|b\| .
$$

Единицей в $A(G)$ является $[G]$. Очевидно, что $\|[G]\|=1$. Предложение доказано.

Коммутативная унитальная банахова алгебра $\widehat{A}(G)$ определяется как пополнение $A(G)$ по норме $\|\cdot\|$. Она состоит из всех таких бесконечных сумм $\widehat{a}=\sum c_{i}\left[H_{i}\right]$, что ряд $\sum\left|c_{i}\right| r\left(\left[H_{i}\right]\right)$ сходится (к норме $\left.\|\widehat{a}\|\right)$. 


\section{Список литературы}

1. Semenov Yu.S. Rings associated with hyperbolic groups // Comm. Algebra. 1994 . V. 22 (15). P. 6323-6347.

2. Neumann $W$. On intersections of finitely generated subgroups of free groups // Lecture Notes in Math. 1990. V. 1456. P. 161-170.

3. Neumann $H$. On intersections of finitely generated subgroups of free groups // Publ. Math. Debrecen. 1955-56. V. 4. P. 186-189; 1957-58. V. 5. P. 128.

Московский государственнњй

Поступила в редакцию

университет путей сообщения

16.01 .1996 